INSTITUT NATIONAL DE RECHERCHE EN INFORMATIQUE ET EN AUTOMATIQUE

\title{
M/D/1/0 loss system with interference and applications to transmit-only sensor networks
}

\author{
Bartłomiej Błaszczyszyn — Božidar Radunović
}

\section{$\mathrm{N}^{\circ} 6073$}

December 2006

Thème COM

\section{apport}

de recherche 



\title{
$\mathrm{M} / \mathrm{D} / \mathbf{1} / 0$ loss system with interference and applications to transmit-only sensor networks
}

\author{
Bartłomiej Błaszczyszyn田, Božidar Radunoviđ \\ Thème COM — Systèmes communicants \\ Projets TREC \\ Rapport de recherche $n^{\circ} 6073$ - December 2006 - 17pages
}

\begin{abstract}
We propose and analyze a probabilistic model of packet reception in the steady state regime of a non-slotted wireless communication channel. It is an extension of the classical M/D/1/0 Erlang's loss model where the interference created by different packet emissions is introduced by means of a shot-noise process. More precisely, we assume that a given packet is admitted by the single receiver if this latter is idle at the packet arrival epoch and successfully received if, in addition, its signal-to-interference-and-noise ratio averaged over the reception period is large enough. As the main result we prove an analog of the Erlang's formula for the ergodic rate of the successfully received packets. Our work is motivated by some applications to transmit-only sensor networks.
\end{abstract}

Key-words: Erlang's loss system, shot-noise process, SINR, stochastic geometry, sensor network

* INRIA \& ENS and Mathematical Institute University of Wrocław, 45 rue d'Ulm, 75005 Paris FRANCE, Bartek.Blaszczyszyn@ens.fr

$\dagger$ Microsoft Research, 7 JJ Thomson Avenue, Cambridge, CB3 0FB, UK. bozidar@microsoft.com. This work was done while the author was a post-doc at INRIA \& ENS, Paris, France.

Unité de recherche INRIA Rocquencourt

Domaine de Voluceau, Rocquencourt, BP 105, 78153 Le Chesnay Cedex (France)

Téléphone : +33139635511- Télécopie : +33139635330 


\section{Système $\mathrm{M} / \mathrm{D} / \mathbf{1} / 0$ avec pertes et interférence avec applications aux réseaux de capteurs sans rétroaction}

Résumé : Nous proposons et analysons un modèle probabiliste de réceptions dans le régime stationnaire d'un canal de communication sans fil non-slotté. C'est une extension du modèle classique d'Erlang avec pertes $\mathrm{M} / \mathrm{D} / 1 / 0$, où l'interférence créée par les émissions de différents paquets est introduite par l'intermédiaire d'un processus de shot-noise. Plus précisément, nous supposons qu'un paquet donné est admis par un récepteur unique si celuici est libre à l'arrivé du paquet et correctement reçu si, en plus, le rapport, moyenné sur la période de réception, du signal sur bruit et interférence est suffisamment grand. Notre résultat principal est une formule analogue à celle d'Erlang, qui donne le taux ergodique du nombre de paquets correctement reçus par le récepteur. Notre travail est motivé par certaines applications aux réseaux de capteurs.

Mots-clés : modèle d'Erlang avec pertes, processus de shot-noise, rapport signal sur bruit, géométrie aléatoire, réseau de capteurs 


\section{Introduction}

Consider packets of some constant length $B$ arriving to a single receiver according to a Poisson point process with constant intensity $\lambda$. Suppose that the receiver can receive at most one packet at a time and that packets arriving when the receiver is busy are simply dropped. The above description corresponds to the $M / D / 1 / 0$ loss model. One knows (from the loss Erlang's formula that applies to the case with a general, in particular deterministic, service time; see e.g. [1 equation (81), p. 71]) that the fraction of packets that are not dropped by this system in the long run is equal to

$$
\frac{1}{1+\lambda B},
$$

where $\lambda B$ is the mean number of arrivals per packet duration.

The above classical loss model is adapted to the situation when there is no interference between signals carrying packets; i.e., when reception of a given packet cannot be damaged by concurring emissions of other packets. If it is not the case, some packets, which are not dropped may not be successfully received due to the interference with other (dropped) packets. A simple model of interference assumes that any two packets collide with each other if their reception periods overlap, and that none of the colliding packets can be successfully received. It is easy to see that in this model the fraction of non-colliding packets is equal to $e^{-2 \lambda B}<1 /(1+\lambda B)$ (cf a non-slotted Aloha model in [2] (4.13)]).

Collisions, as a model of interference is not adequate in many situations either. In particular in radio communications, when signals caring packets arrive with different powers, a weak single interfering transmission may not prevent a much stronger signal from being received, but many weak interfering signals may jointly do it. A more detailed packet reception model, taking into account particular system assumptions, is then required.

In this paper we present and analyze some extension of the classical loss model $M / D / 1 / 0$, where the interference between packet emissions is taken into account by means of the so called shot-noise model. We assume that a given packet is successfully received if its signalto-interference-and-noise ratio (SINR) averaged over the reception period is large enough. As the main result we prove an analog of the Erlang's formula for the ergodic rate of the packets that are successfully received. The expression is explicit in the case of exponential received powers, which is a valid assumption e.g. in the presence of the Rayleigh fading.

We discuss all basic system assumptions of our model and apply it to the analysis of the event-to-sink performance of in some transmit-only sensor network (cf. [3] for standard sensor networks).

The remaining part of this paper is organized as follows. In Section 2 we give general physical and protocol assumptions for our moder. In Section 3 we present the model and prove the main result. In Section 4 the model is extended by introducing locations of emitters on the plane. Applications to sensor networks are presented in Section 5 Some conclusions and future works are signaled in Section [6] 


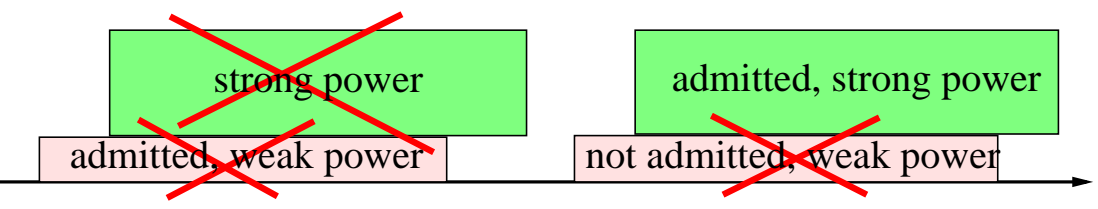

Figure 1: Gain due to admission policy.

\section{Model assumptions}

Now, we will describe briefly basic system assumptions which lead to our model. A motivating example of transmit-only sensor network is presented in Section 5.1

\subsection{Reception policy}

Consider a non-slotted wireless channel in which packets of a fixed duration arrive in time at a single receiver. The receiver needs to synchronize to a packet before receiving it. Once synchronized to a packet, the receiver starts receiving it and continues until the end of the packet transmission. If the transmission is lost because of the interference with other packet emissions (see the signal to interference ratio condition (2.1) below), the error will be detected only at the end of the reception. Moreover, all the interfering packets will be lost as well. In order to improve the reception efficiency, one may consider an additional packet admission (thinning) policy. It consists in letting the receiver, once it is synchronized to a packet, to decide whether it starts receiving or ignore the detected packet. Such a policy, based for example on the value of the instantaneous received power or some average of the power received previously from a given emitter, may allow to ignore some too weak packets and to make the receiver more often available for stronger packets; cf. Figure 1 The choice of a particular policy depends on system design goals. We will consider some examples in Section 5

\subsection{SINR condition}

We suppose that a packet is successfully received if the SINR empirically averaged over the reception duration, is higher than some threshold. Otherwise, the packet is lost. When doing this, we have in mind a slow fading Gaussian channel model in which each bit is sent through many symbols (like in repetition coding, UWB spreading or CDMA) with interleaving. By this latter we mean that the symbols corresponding to a given bit are uniformly distributed over the duration of the packet size. Suppose that the receiver is equipped with a matched filter (coherent maximal ratio combiner). Then, the standard analysis (see e.g. 4, Section 3.2.1]) says, that the link quality corresponds to some threshold $\gamma$ on the SINR

$$
\frac{H P}{W+1 / M \sum_{j=1}^{M} I_{j}} \geq \gamma,
$$


where $M$ is the number of symbols in the packet, $W$ is the noise power, $I_{j}$ is the interfering power received at $j$ th symbol, $P$ is the received power averaged over fading effects that is supposed to depend only on the emitted power and the distance between the emitter and the receiver, $H$ is the value of the fading and/or scattering in the link from the emitter to the receiver; it is assumed to be constant during the packet reception. Remark that it is the interleaving that allows for the empirical averaging of the interference $\left\{I_{j}\right\}$ in 2.1) over the packet duration. We will interpret (2.1) as the SINR condition identifying the successful reception of the packet.

\subsection{Rayleigh fading}

Rayleigh fading is a commonly used fading model that consists in assuming that the real and complex components of the baseband representation of the received signal are multiplied by independent Gaussian random variable. This corresponds to the multiplication of the power of the received (baseband) signal by an exponential random variable $H$ (see e.g. [4, p. 50 and 501]). We will assume this model throughout the whole paper.

\section{$3 \mathrm{M} / \mathrm{D} / 1 / 0$ loss system with interference}

Now we present our loss model for the reception of packets in the non-slotted wireless communication channel with interference, whose assumptions are described the previous section. We use a queuing theory approach to model arrivals of packets in time. The service discipline is similar to this of the Erlang's loss system with one server and no waiting room, with a modification that takes into account the SINR condition (2.1).

\subsection{The model}

Assume a time homogeneous, independently marked Poisson point process

$$
\Phi=\left\{\left(T_{n},\left(P_{n}, H_{n}\right)\right)\right\}_{n=-\infty}^{\infty},
$$

where $T_{n}$ are customer (packet) arrival epochs and $\left(P_{n}, H_{n}\right)$ are independent, identically distributed (i.i.d.) marks, where $P_{n} \geq 0, H_{n} \geq 0$ can be interpreted as, respectively, the average (over fading effects) power with which the $n$th packet is received and the actual fading state of its channel. 1 In this paper we will always assume that $P_{n}$ and $H_{n}$ are independent of each other and $H_{n}$ are exponentially distributed with parameter 1 . Lets denote by $\lambda(0<\lambda<\infty)$ the intensity of $\Phi$; i.e., $T_{n+1}-T_{n}$ are i.i.d. exponential random variables (r.v.) with parameter $\lambda$.

\footnotetext{
${ }^{1}$ The randomness of $\left\{P_{n}\right\}$ can reflect different locations of transmitters and/or powers with which they emit packets, while the randomness of $\left\{H_{n}\right\}$, given fixed emitter and receiver, reflects the temporal variation of the channel conditions.
}

$\mathrm{RR} \mathrm{n}^{\circ} 6073$ 
We consider the following modification of the Erlang's loss policy. Suppose that each packet is admitted by the single server of the system (i.e., starts being received by the receiver) if this latter is idle at the packet's arrival epoch and dropped (rejected) otherwise. Admitted packets are being received during their duration time $B$. However, dropped packets interfere during their emissions with the packets that are being received. Inspired by inequality (2.1), we will say that the $n$th packet, given it is admitted by the receiver, is successfully received if the following inequality holds

$$
\frac{P_{n} H_{n}}{W+1 / B \int_{T_{n}}^{T_{n}+B} I(t) \mathrm{d} t-P_{n} H_{n}} \geq \gamma,
$$

where $W$ is some nonnegative r.v. independent of $\Phi, \gamma>0$ is some constant, $I(t)$ is the value of the following temporal shot-nose process at time $t$

$$
I(t)=\sum_{i=-\infty}^{\infty} H_{i} P_{i} \mathbf{1}_{[0, B)}\left(t-T_{i}\right)
$$

describing the total power received at time $t$ from all packets that are being sent (including the power of the packet that is being received; this is why we subtract $P_{n} H_{n}$ from $I(t)$ in (3.1).

\subsection{Rate of successful reception}

Our goal is to calculate the ergodic rate of successful reception of packets; i.e.,

$$
\pi=\lim _{N \rightarrow \infty} \frac{1}{N} \#\{\text { packets successfully received from } n=1, \ldots, N\},
$$

where \# denotes the cardinal number. Denote by $\zeta(t)$ the indicator of the event that the receiver is busy at time $t$ and $\zeta(0-)=\lim _{t \uparrow 0} \zeta(t)$. Denote by $\delta_{n}$ the indicator of the event that the inequality (3.1) holds. Let $\mathbf{P}^{0}$ denote the Palm probability given there is a customer arrival at time 0 and let $\mathbf{E}^{0}$ denote the corresponding expectation. By Slivnyak's theorem, under $\mathbf{P}^{0}$ arrivals form the original stationary Poisson point process with an extra arrival $\left(T_{0},\left(P_{0}, H_{0}\right)\right)$ added at time $T_{0}=0$, whose mark is independent of everything else and originally distributed. The following result is a consequence of the ergodic theorem.

Proposition 3.1 The limit defining $\pi$ exists $\mathbf{P}^{0}$ almost surely and $\pi=\mathbf{E}^{0}\left[(1-\zeta(0-)) \delta_{0}\right]$.

Proof: Our system is ergodic because it is driven by independently marked Poisson point process $\Phi$, and the result follows form the ergodic theorem (see e.g. [5. Theorem 1.6.1]).

In order to express $\pi$ more explicitly we need the following notation. Let $\mathcal{L}_{W}(\xi)$ be the Laplace transform of $W$ and define

$$
\begin{aligned}
& \mathcal{L}_{1}(\xi)=\exp \left(-\lambda B\left(1-\mathbf{E}\left[\frac{1}{\xi P} \log (1+\xi P)\right]\right)\right), \\
& \mathcal{L}_{2}(\xi)=\exp (-\lambda B)\left(1+\lambda B \int_{0}^{1} \exp \left(\lambda B \mathbf{E}\left[\frac{1}{\xi P} \log (1+\xi P t)\right]\right) \mathrm{d} t\right) .
\end{aligned}
$$




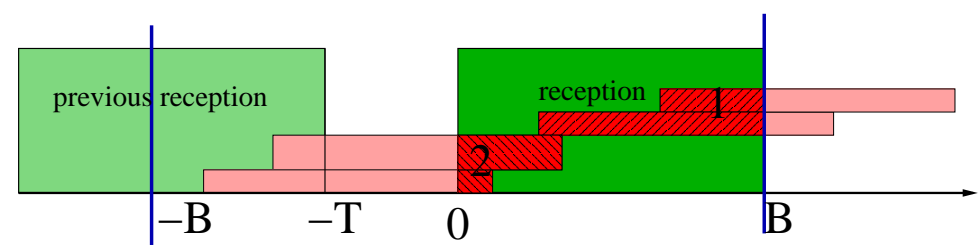

Figure 2: Components of interference $I^{\prime}(t)$.

Remark: Note that the packets which contribute to

$$
I^{\prime}(t)=I(t)-P_{0} H_{0}=\sum_{i \neq 0}^{\infty} H_{i} P_{i} \mathbf{1}_{[0, B)}\left(t-T_{i}\right)
$$

during the period $t \in\left[0, B\right.$ ) (which, under Palm distribution $\mathbf{P}^{0}$, is the reception period of the packet $\left(T_{0}=0,\left(P_{0}, H_{0}\right)\right)$, provided it is admitted) can arrive only in $(-B,-T] \cup(0, B)$, where $-T$ is the end of the last busy period before time 0 (see Figure 2). We will see later that $\mathcal{L}_{1}, \mathcal{L}_{2}$ are Laplace transforms of the contributions made to $1 / B \int_{0}^{B} I^{\prime}(t) \mathrm{d} t$ by the packets arriving, respectively in $(0, B)$ and $(-B,-T]$.

Now we give the main result of this Section - an Erlang's type formula.

Proposition 3.2 Consider the Erlang's M/D/1/0 loss system with interference described in Section 3.1. Then

$$
\pi=\frac{1}{1+\lambda B} \mathbf{E}\left[\mathcal{L}_{W}\left(\gamma / P_{0}\right) \mathcal{L}_{1}\left(\gamma / P_{0}\right) \mathcal{L}_{2}\left(\gamma / P_{0}\right)\right]
$$

where the expectation is taken with respect to the random variable $P_{0}$.

Proof: By Proposition $3.1 \pi$ is equal to

$$
\begin{aligned}
\mathbf{E}^{0}\left[(1-\zeta(0-)) \delta_{0}\right] & =\mathbf{P}^{0}\{\zeta(0-)=0\} \mathbf{E}^{0}\left[\delta_{0} \mid \zeta(0-)=0\right] \\
& =\mathbf{P}^{0}\{\zeta(0-)=0\} \mathbf{P}^{0}\left\{H_{0} \geq \gamma\left(W+I_{B}^{\prime}\right) / P_{0} \mid \zeta(0-)=0\right\}
\end{aligned}
$$

where $I_{B}^{\prime}=1 / B \int_{0}^{B} I^{\prime}(t) \mathrm{d} t$. By the Erlang's loss formula (cf. formula (1.1)) $\mathbf{P}^{0}\{\zeta(0-)=$ $0\}=1 /(1+\lambda B)$. Moreover, $\mathbf{P}^{0}\{\cdot \mid \zeta(0-)=0\}$ corresponds to the Palm probability $\mathbf{P}_{a}^{0}$ given the packet arriving at time 0 is accepted by the receiver (this can be proved formally e.g. by the Neveu exchange formula [5] Section 1.3.2]). Consequently, using the exponential tail of $H_{0}$ and the fact that it is independent of everything else, we have

$$
\pi=\frac{1}{1+\lambda B} \mathbf{E}_{a}^{0}\left[e^{-\gamma\left(W+I_{B}^{\prime}\right) / P_{0}}\right] .
$$

Conditioning on $P_{0}$ and noting that $W$ is independent of $I_{B}^{\prime}$ we obtain

$$
\pi=\frac{1}{1+\lambda B} \mathbf{E}\left[\mathcal{L}_{W}\left(\gamma / P_{0}\right) \mathbf{E}_{a}^{0}\left[e^{-\gamma I_{B}^{\prime} / P_{0}} \mid P_{0}\right]\right]
$$




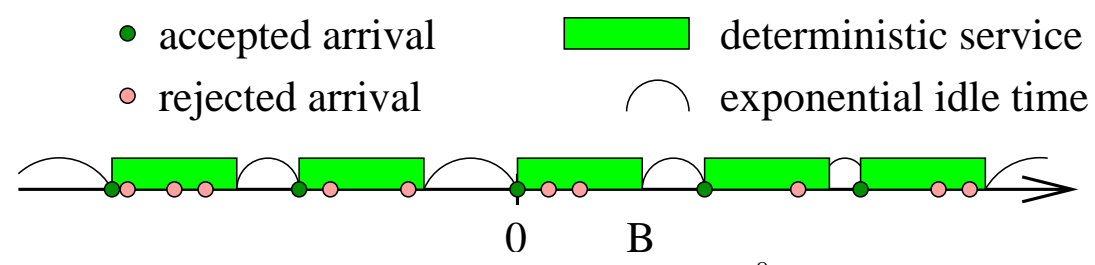

Figure 3: Arrivals under $\mathbf{E}_{a}^{0}$.

Note that the integrated shot-noise is also a "shot-noise type" variable. Indeed, by Fubini theorem $I_{B}^{\prime}=1 / B \int_{0}^{B} \sum_{i \neq 0} H_{i} P_{i} \mathbf{1}_{[0, B)}\left(t-T_{i}\right)=\sum_{i \neq 0} H_{i} P_{i} V\left(T_{i}\right)$, where $V(t)$ is equal to $B-|t|$ for $|t| \leq B$ and 0 elsewhere. Denote

$$
\begin{aligned}
I_{B}^{1} & =\sum_{i \neq 0} H_{i} P_{i} V\left(T_{i}\right) \mathbf{1}_{(0, B)}\left(T_{i}\right), \\
I_{B}^{2} & =\sum_{i \neq 0} H_{i} P_{i} V\left(T_{i}\right) \mathbf{1}_{(-B,-T]}\left(T_{i}\right),
\end{aligned}
$$

where $-T$ is the end of the last busy period before time 0 (cf Figure 2 and the Remark after Proposition 3.2). We obviously have $I_{B}^{\prime}=I_{B}^{1}+I_{B}^{2}$. In order to prove the result it remains to show that under $\mathbf{P}_{a}^{0}$ the variables $I_{B}^{1}, I_{B}^{2}$ are independent with their Laplace transforms given by (3.3), (3.4), respectively. This is a consequence of the following fact.

Lemma 3.3 Under $\mathbf{P}_{a}^{0}$ (i.e., given a packet arriving at time 0 is accepted by the receiver)

- the process of accepted arrivals

$$
\Phi_{a}=\left\{\left(T_{n},\left(P_{n}, H_{n}\right)\right): \zeta\left(T_{n}-\right)=0\right\}_{n=-\infty}^{\infty}=\left\{\left(T_{n}^{a},\left(P_{n}^{a}, H_{n}^{a}\right)\right)\right\}_{n=-\infty}^{\infty}
$$

is an independently marked zero-delayed (i.e., $T_{0}^{a}=0$ ) renewal process with the generic inter-arrival time equal to $B+\mathcal{E}$, where $\mathcal{E}$ is exponential with parameter $\lambda$,

- the process of the arrivals that are rejected

$$
\Phi_{r}=\left\{\left(T_{n},\left(P_{n}, H_{n}\right)\right): \zeta\left(T_{n}-\right)=1\right\}_{n=-\infty}^{\infty}
$$

is an independent marked, double stochastic Poisson point process with intensity equal to $\lambda$ on the set $\bigcup_{n}\left(T_{n}^{a}, T_{n}^{a}+B\right]$ and 0 elsewhere.

Note by Lemma 3.3 (cf Figure 31) that under $\mathbf{E}_{a}^{0}$ and given accepted arrivals, the variables $I_{B}^{2}$ and $I_{B}^{1}$ are independent, as driven by Poisson arrivals in disjoint subsets $(-B,-T]$ and $(0, B)$. Moreover, the (rejected) arrivals in $(0, B)$ form independently marked Poisson point process. Thus, applying the general formula for its Laplace transform (see e.g. [6])) we get

$$
\mathbf{E}_{a}^{0}\left[e^{-\xi I_{B}^{1}}\right]=\exp \left(-\lambda \int_{0}^{B}\left(1-\mathbf{E}\left[e^{-\xi H P V(t)}\right]\right) \mathrm{d} t\right),
$$


where $H, P$ are independent, generic r.v's for $\left\{H_{n}\right\}$ and $\left\{P_{n}\right\}$. Integrating with respect to $t$ and evaluating the expectation with respect to the exponential r.v. $H$ we obtain $\mathbf{E}_{a}^{0}\left[e^{-\xi I_{B}^{1}}\right]=\mathcal{L}_{1}(\xi)$.

In order to calculate $\mathbf{E}_{a}^{0}\left[e^{-\xi I_{B}^{2}}\right]$, we condition on $T$, which by Lemma 3.3$]$ is exponential r.v. with parameter 1 , and we use similar arguments, with integral $\int_{0}^{B}$ in (3.6) replaced be $\int_{-B}^{-T}$ (note that this integral is null if $T>B$ ). Similarly as for $I_{B}^{1}$ and then by integration with respect to the law of exponential $T$ we obtain $\mathbf{E}_{a}^{0}\left[e^{-\xi I_{B}^{2}}\right]=\mathcal{L}_{2}(\xi)$, which completes the proof.

Proof:[of Lemma 3.3 It is easy to see that the process of accepted arrivals is a renewal process with the generic renewal time equal to $B+\mathcal{E}$, where $\mathcal{E}$ is the residual inter-arrival time of $\Phi$. By the by the lack of memory property of the exponential r.v., $\mathcal{E}$ is exponential with parameter $\lambda$. The first statement follows then from the corresponding result for the renewal process.

The second statement follows from the strong Markov property of the Poisson point process since the realization of $\Phi_{a}$ is invariant with respect to any modification of the points of $\Phi$ in $\bigcup_{n}\left(T_{n}^{a}, T_{n}^{a}+B\right]$ (see e.g. [7]).

\subsection{External interference}

Let us introduce now to the Erlang's loss model an additional (external) stationary, ergodic process $J(t)$ of interference, independent of $W$ and $\Phi$. (For example, in Section 4 we will define $J(t)$ as the interference created by some emitters transmitting packets that are not supposed to be received by our receiver at all.) Lets say that the $n$th packet of $\Phi$, given it is admitted by the receiver, is successfully received if the inequality (3.1) holds with the term $1 / B \int_{T_{n}}^{T_{n}+B} J(t) \mathrm{d} t$ added to the denominator. Denote by $\delta_{n}^{\prime}$ the indicator of this event and $\pi^{\prime}=\mathbf{E}^{0}\left[(1-\zeta(0-)) \delta_{0}^{\prime}\right]$. Denote by $J_{B}=\int_{0}^{B} J(t) \mathrm{d} t$ and its Laplace transform by $\mathcal{L}_{J_{B}}(\xi)=\mathbf{E}\left[e^{-\xi J_{B}}\right]$. We have the following straightforward extension the Proposition 3.2

Corollary 3.4 Consider the Erlang's M/D/1/0 loss system with external interference $J(t)$ independent of $\Phi$ and $W$. Under the same other assumptions as in Proposition 3.2 we have

$$
\pi^{\prime}=\frac{\mathbf{E}\left[\mathcal{L}_{W}\left(\gamma / P_{0}\right) \mathcal{L}_{1}\left(\gamma / P_{0}\right) \mathcal{L}_{2}\left(\gamma / P_{0}\right) \mathcal{L}_{J_{B}}\left(\gamma / P_{0}\right)\right]}{1+\lambda B} .
$$

\section{Planar extensions of the loss system}

In this section we assume that packets are emitted from different locations of the plane $\mathbb{R}^{2}$ and arrive (at the epochs of a temporal Poisson process) at the receiver that operates according to the model described in Section 3.1 Given locations of the emitters and the receiver, and assuming some form of the power attenuation function, we will obtain a particular form of the distribution of the received powers $\left\{P_{n}\right\}$. Note that this distribution was not specified in the previous section. We will also consider some packet pre-filtering policy 
(thinning) applied by the receiver: only a fraction of the packets emitted from a given location is supposed to be captured by the receiver. Packets that are rejected by this thinning policy create the external interference $J(t)$ considered in Section 3.3 More precisely, we introduce the following notions.

Attenuation function Suppose that the signal transmitted with some power $\bar{P}$ from the location $x$ is attenuated (on average over fading effects) on the path to the receiver located at 0 by the factor $L(x)>0$; i.e., the ergodic mean of the power received at 0 is equal to $\bar{P} L(x)$.

Spatial thinning of packets Suppose that packets are emitted from different locations of the plane $\mathbb{R}^{2}$. Given a function $0 \leq d(x) \leq 1$ of $x \in \mathbb{R}^{2}$, consider the following spatial thinning policy: with probability $d(x)$, independently of everything else, the receiver considers a given packet emitted from $x$ as admissible and starts receiving it, provided it is idle. Otherwise, the receiver ignores (drops) the packet even if it is idle at its arrival epoch.

\subsection{Poisson rain of packets}

Consider a spatio-temporal Poisson process $\left\{\left(X_{n}, T_{n}\right)\right\}$ where $X_{n} \in \mathbb{D}$ for some $\mathbb{D} \subseteq \mathbb{R}^{2}$, $T_{n} \in \mathbb{R}$, with intensity measure $\Lambda(\mathrm{d} x) \times \lambda_{e} \mathrm{~d} t$. The coordinates of the point $\left(X_{n}, T_{n}\right)$ denote, respectively, the location form which the packet is emitted and the time the emission starts. (One can think of emitters being born at locations $X_{n}$ and time $T_{n}$ just to emit one packet at this moment; after the transmission of this packet the emitter disappears.) We assume that the points $\left\{\left(X_{n}, T_{n}\right)\right\}$ are independently marked by i.i.d. exponential (with mean 1 ) random variables $H_{n}$ modeling the fading conditions during the transmission $n$. Moreover, assuming some thinning policy $d(\cdot)$, we suppose that the points are further marked by i.i.d Bernoulli r.v.s $U_{n}$ describing the admissibility status of the corresponding packets; i.e., $\mathbf{P}\left\{U_{n}=1\right\}=1-\mathbf{P}\left\{U_{n}=0\right\}=d\left(X_{n}\right)$, where $U_{n}=1$ marks an admissible packet.

We call the marked Poisson point process

$$
\Psi=\Psi_{d}=\left\{\left(\left(X_{n}, T_{n}\right),\left(H_{n}, U_{n}\right)\right)\right\}_{n},
$$

the Poisson rain of arrivals with a given spatial thinning policy $d(\cdot)$. We consider $\Psi$ as driving the Erlang's loss system with interference described in Section 3 in the following sense. We define $P_{n}=\bar{P} L\left(X_{n}\right)$ and take the admissible packet transmissions $\Phi=\left\{\left(T_{n},\left(P_{n}, H_{n}\right)\right)\right.$ : $\left.U_{n}=1\right\}_{n}$ as the input to the system. The total received power from packets that are not admissible

$$
J(t)=\sum_{n}\left(1-U_{n}\right) H_{n} \bar{P} L\left(X_{n}\right) \mathbf{1}_{[0, B)}\left(t-T_{n}\right),
$$

is considered as the external interference (cf Section 3.3).

Denote by

$$
\lambda=\lambda_{e} \int d(x) \Lambda(\mathrm{d} x)
$$




$$
\begin{aligned}
\mathcal{L}_{1}(\xi) & =\exp \left(-\lambda B+\lambda_{e} B \int \frac{d(x)}{\xi \bar{P} L(x)} \log (1+\xi \bar{P} L(x)) \Lambda(\mathrm{d} x)\right) \\
\mathcal{L}_{2}(\xi) & =\exp (-\lambda B)\left(1+\lambda B \int_{0}^{1} \exp \left(\lambda_{e} B \int \frac{d(x)}{\xi \bar{P} L(x)} \log (1+\xi \bar{P} L(x) t) \Lambda(\mathrm{d} x)\right) \mathrm{d} t\right) \\
\mathcal{L}_{J_{B}}(\xi) & =\exp \left(-2 \lambda_{e} B \int(1-d(x))\left(1-\frac{1}{\xi \bar{P} L(x)} \log (1+\xi \bar{P} L(x))\right) \Lambda(\mathrm{d} x)\right) \\
\mathcal{L}(\xi) & =\exp \left(-\lambda_{e} B \int\left(1-\frac{1}{\xi \bar{P} L(x)} \log (1+\xi \bar{P} L(x)) \Lambda(\mathrm{d} x)\right)\right)
\end{aligned}
$$

the temporal intensity of Poisson process of the packets admissible according to the spatial thinning $d(\cdot)$. In what follows we always assume that $0<\lambda<\infty$.

The following consequence of Corollary 3.4 gives the Erlang's type formula for the Poisson rain model.

Corollary 4.1 Consider the Erlang's loss system $M / D / 1 / 0$ driven by the Poisson rain of events $\Psi$ in some domain $\mathbb{D} \times \mathbb{R}$ with spatial admission policy $d(\cdot)$. Then, the fraction $\pi^{\prime}=\pi^{\prime}(x)$ of admissible packets successfully received from a location $x \in \mathbb{R}^{2}$, given there is an emitter located there, is given by Corollary [3.4 with constant $P_{0}=\bar{P} L(x)$, and $\mathcal{L}_{1}, \mathcal{L}_{2}, \mathcal{L}_{J_{B}}$ given, respectively, by (4.3), (4.4), 4.5), where $\lambda$ is given by (4.2) and the integrals are taken over $\mathbb{D}$, provided $\lambda<\infty$.

Proof: Note that the distribution of the mean received power is equal to

$$
\mathbf{P}\{P \leq a\}=\frac{\int d(x) \mathbf{1}(\bar{P} L(x) \leq a) \Lambda(\mathrm{d} x)}{\int d(y) \Lambda(\mathrm{d} y)},
$$

which is correctly defined since we assume $\lambda<\infty$. Then, formulas (4.3), (4.4) follow, respectively, from (3.3), (3.4). Next, note that $\Phi$ and $J(t)$ are independent; this is a consequence of the independent thinning of the Poisson process of all packet emissions. Moreover, the integrated interference $J_{B}$, given by the formula $\left.J_{B}=\sum_{n}\left(1-U_{n}\right)\right) H_{n} \bar{P} L\left(X_{n}\right) V\left(T_{n}\right)$ is a shot-noise type random variable (cf. the proof of Proposition 3.2). Its Laplace transform $\mathcal{L}_{J_{B}}(\xi)$ is known explicitly and can be simplified to (4.5).

Remark: Fixed emitters located at $\left\{X_{i}\right\}$, each sending packets with some temporal Poisson process, can be seen as a special case of the Poisson-rain of packets, with purely atomic spatial density measure $\Lambda(D)=\#\left\{X_{i}: X_{i} \in D\right\}$. Then the integrals $\int(\ldots) \mathrm{d} x$ in formulas (4.3)- (4.5) take form of the respective sums $\sum_{X_{i}}(\ldots)$. Moreover, if the spatial repartition of $X_{i}$ is sufficiently dense, then these atomic measures can be reasonably "smoothed out" leading to approximative integral formulas. 


\subsection{Bounds}

In this section we will give some simple bounds for the rate of successful reception of packets. Denote $\gamma_{x}=\gamma /(\bar{P} L(x))$.

Corollary 4.2 Under the assumptions of Corollary 4.1. we have

$$
\frac{\mathcal{L}_{W}\left(\gamma_{x}\right)\left(\mathcal{L}\left(\gamma_{x}\right)\right)^{2}}{1+\lambda B} \leq \pi^{\prime}(x) \leq \frac{\mathcal{L}_{W}\left(\gamma_{x}\right) \mathcal{L}\left(\gamma_{x}\right)}{1+\lambda B}
$$

where $\lambda$ and $\mathcal{L}$ are given by (4.2) and (4.6), respectively.

Proof: Note first that $\mathcal{L}_{2}(\xi) \geq \mathcal{L}_{1}(\xi)$. This can be verified directly comparing formulas (3.3) and (3.4), but a simple probabilistic argument can be used as well. Recall that $\mathcal{L}_{1}(\xi)$ is the Laplace transform of $I_{B}^{1}$ given by (3.4), whereas $\mathcal{L}_{2}(\xi)$ is the Laplace transform of $I_{B}^{2}$ given by (3.5). Than, the lower bound of Corollary 4.2 follows immediately from (4.3) and (4.5). In order to get the upper bound, it is enough to observe that $\mathcal{L}_{2} \leq 1$ and to take $\mathcal{L}_{J_{B}}$ with factor 2 in the exponent of (4.5) replaced by 1.

Remark: The upper bound in Corollary 4.2 consists in taking no interfering arrivals before reception of a given packet, whereas the lower bound consists in assuming the unconditional Poisson process of such arrivals ( $T=0$ in the proof of Proposition 3.2).

\section{Applications to sensor networks}

Will show now how the results presented in previous sections can by used to analyze and optimize the performance of the sensors-to-cluster-heads information transport in some transmitonly sensor network.

\subsection{Transmit only sensor network}

Let us consider a network of sensors and cluster-heads. Sensors are simple sensing devices that are equipped only with a single transmitter. They send information to the cluster-heads without acknowledgments (blindly). More precisely, we assume that some events trigger transmissions at the sensors randomly and independently of each other, with some constant intensity. Cluster-heads are more powerful (and more expensive) sensors. They are equipped with a receiver, and their special role is to receive information from transmit-only sensors. The synchronization, reception and decoding assumptions concerning these transmissions are described in Section 2 2 The network consists of a large number of sensors and a much smaller number of cluster-heads. We want to analyze and optimize the performance of the information transport from sensors to cluster-heads.

\footnotetext{
${ }^{2}$ We tacitly assume also that cluster-heads have a reliable communication channel of a higher rate used to forward the collected information to a central server, and that this channel does not interfere with the sensor channel.
} 


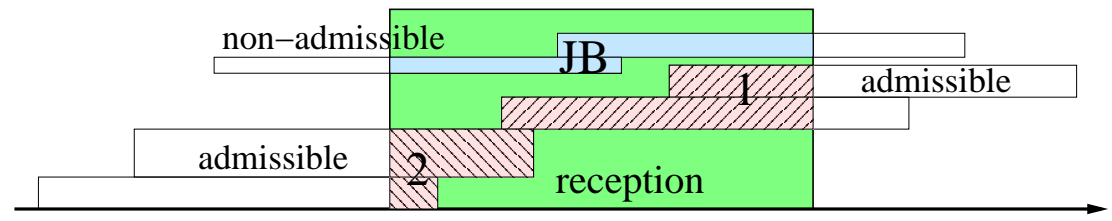

Figure 4: Three terms of interference $\mathcal{L}_{1}, \mathcal{L}_{2}, \mathcal{L}_{J_{B}}$.

In what follows, for simplicity, we assume one only one cluster located at the origin of the plane. Sensors in a given "real" network realization are fixed. Our previous results let us evaluate the performance of a given fixed configuration of sensors served by the cluster head applying some spatial thinning policy (cf the Remark after the proof of Corollary 4.1). However, for performance optimization it is more convenient to use the Poisson-rain approximation (cf Section 4.1). It is a reasonable approximation of the packet traffic generated be an arbitrary (also deterministic) repartition of sensors on the plane, provided the density of sensors is large (cf the Remark after the proof of the Corollary 4.1).

\subsection{Density of received information}

Our principal performance metric is the spatio-temporal density $\rho(x)$ of collected information. We define it as the mean number of packets received from sensors, per second, from the surface area $\mathrm{d} x$.

Assume for simplicity a homogeneous Poisson rain of packets with intensity $\Lambda(\mathrm{d} x)=\lambda_{s} \mathrm{~d} x$ and a spatial thinning policy $d(x)$. Denote by $\lambda=\lambda_{e} \int d(r) \lambda_{s} \mathrm{~d} x$ the total intensity of the admissible packets and let $\gamma_{x}=\gamma /(\bar{P} L(x))$, where $\bar{P}$ is the emission power used be all sensors, and $L(x)$ is the power attenuation function. The following fact follows from the Campbell's formula and Corollary 4.1

Corollary 5.1 The density of received information is equal to $\rho(x)=\lambda_{e} \lambda_{s} d(x) p_{\text {free }} p_{\text {rec }}(x)$, where $p_{\text {free }}=1 /(1+\lambda B)$ is the probability that a typical admissible packet finds the cluster head idle when it arrives and $p_{\text {rec }}(x)=\mathcal{L}_{W}\left(\gamma_{x}\right) \mathcal{L}_{1}\left(\gamma_{x}\right) \mathcal{L}_{2}\left(\gamma_{x}\right) \mathcal{L}_{J_{B}}\left(\gamma_{x}\right)$ is the conditional probability of its successful reception.

Recall that $\mathcal{L}_{1}, \mathcal{L}_{2}, \mathcal{L}_{J_{B}}$ are the Laplace transforms of the interference averaged over the reception period, generated respectively, by: admissible packets arriving when it is being received, admissible packets that are being sent at its arrival epoch, all non-admissible packets; cf. Figure 4] These Laplace transforms are explicitly given by formulas (4.3), (4.4), (4.5) with $\Lambda(\mathrm{d} x)=\lambda_{s} \mathrm{~d} x$.

Corollary 4.2 gives two more explicit bounds on $p_{\text {rec }}(x)$.

Corollary 5.2 We have $\underline{p}_{r e c}(x) \leq p_{\text {rec }}(x) \leq \bar{p}_{\text {rec }}(x)$, where $\underline{p}_{r e c}(x)=\mathcal{L}_{W}\left(\gamma_{x}\right) \mathcal{L}^{2}\left(\gamma_{x}\right), \bar{p}_{\text {rec }}(x)=$ $\mathcal{L}_{W}\left(\gamma_{x}\right) \mathcal{L}\left(\gamma_{x}\right)$ and $\mathcal{L}$ is given by $\left.\overline{4.6}\right)$.

Denote by $\rho(x), \bar{\rho}(x)$, respectively, the lower and the upper bound of $\rho(x)$ obtained when $p_{r e c}(x)$ in Corollary 5.1 is replaced by, respectively, $\underline{p}_{r e c}(x)$ and $\bar{p}_{r e c}(x)$. Note that both 
$\underline{p}_{r e c}(x)$ and $\bar{p}_{\text {rec }}(x)$ do not depend on $d(\cdot)$, which makes optimization of $\underline{\rho}(x), \bar{\rho}(x)$ easier (for the quality of these bounds see Figure 5 (left)).

\subsection{Optimization}

We consider two design goals, related to the transport of the information from sensors to the cluster-head. More details can be found in [8].

Transport-aware coverage Knowing that the attenuation function $L(x)$ (and thus $p_{\text {rec }}(x)$ ) typically decreases with the distance $|x|$ to the cluster-head, one may compensate it by some spatial thinning policy $d(x)$. Precisely, one might be interested in finding a policy $d(x)$ which maximizes the constant density $\rho(x)=\rho$ is some given domain $\mathbb{D}$. Alternatively, one might be interested in maximizing the domain $\mathbb{D}$ while providing some minimal density $\rho(x) \geq \rho$. Consider the following example.

Example 5.3 Assume a distance-dependent path-loss $L(x)=L(|x|)$ model. Given radius $R$ of the disk $B(0, R)$ centered at 0 , we are looking for $d(x)$ that maximizes $\rho$ under the constraint $\rho(x) \geq \rho$ for $|x|<R$. To solve this problem, denote

$$
\underline{I}=\underline{I}(R)=\int_{|x|<R} \frac{1}{\underline{p}_{r e c}(x)} \mathrm{d} x
$$

and

$$
\underline{M}=\underline{M}(R)=\max _{|x|<R} \frac{1}{\lambda_{s} \underline{p} \underline{r e c}(x)} .
$$

For a given policy $d(\cdot)$ denote the total spatial intensity of admissible packets by

$$
\|d\|_{\lambda_{s}}=\int d(x) \lambda_{s}(x) \mathrm{d} x
$$

One can prove the following fact (see [8]). The policy

$$
d(x)=d_{R}(x)= \begin{cases}1 /\left(\underline{M} \lambda_{s} \underline{p}_{r e c}(x)\right) & \text { for }|x|<R \\ 0 & \text { for }|x|>R\end{cases}
$$

gives constant $\rho(x)=\rho=1 /\left(B \underline{I}+\underline{M} / \lambda_{e}\right)$ for $|x|<R$. Moreover, under this policy we have $\rho(x) \geq \underline{\rho}$ for $\mid x \bar{x}<R$.

Similarly, the policy that maximizes constant $\bar{\rho}(x)=\bar{\rho}$ for $|x|<R$ can be found and one can show that there is no policy $d(\cdot)$ for which $\rho(x) \geq \bar{\rho},|x|<R$, with the strict inequality on some non-null set $\mathrm{d} x$. 

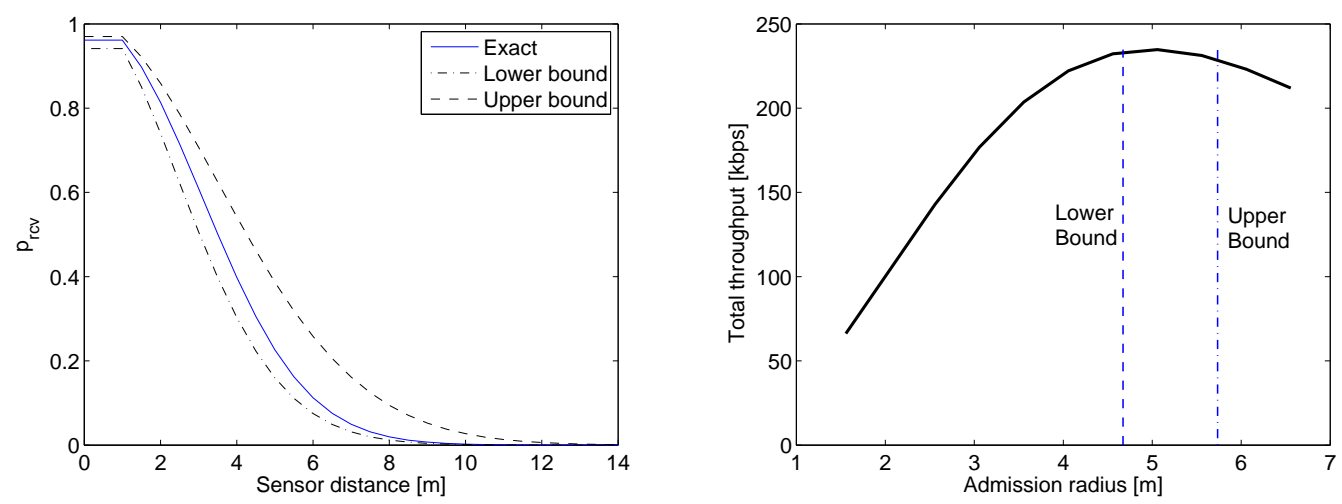

Figure 5: Exact value and approximations of $p_{\text {rec }}$ (left). Maximization of the total intensity of information (right). Numerical values correspond to a channel with the throughput of $1 \mathrm{MB} / \mathrm{s}$ at the physical layer, which is shared by sensors homogeneously distributed with the density of $\lambda_{s}=10$ sensors $/ \mathrm{m}^{2}$, with SINR threshold $\gamma=1$, path-loss model $L(x)=$ $L(|x|)=\kappa|x|^{-\eta}$ with $\kappa=10^{-5.5}, \eta=3.3$.

Total throughput We are interested now in maximization of the total intensity of information

$$
U=\int_{\mathbb{D}} \rho(x) \mathrm{d} x,
$$

received from $\mathbb{D}=\mathbb{R}^{2}$. Denote by $\underline{U}, \bar{U}$, respectively, the lower and the upper bound of the total weighted intensity of information obtained when $\rho(x)$ is replaced by $\underline{\rho}(x)$ and $\bar{\rho}(x)$. As previously, we can solve this global optimization problem for the bounds $\underline{\rho}(x)$ and $\bar{\rho}(x)$, and in this way approximate the solution of the original problem. Consider the following example.

Example 5.4 Assume a distance-dependent path-loss $L(x)=L(|x|)$ model. For $\theta \geq 0$ Denote

$$
R(\theta)=\max \left\{R \geq 0: \lambda_{s} \underline{p}_{r e c}(R) \geq \theta\right\}
$$

and

$$
\underline{\theta}^{*}=\underset{\theta \geq 0}{\arg \max } \frac{\int_{|x|<R(\theta)} \lambda_{s} \underline{p}_{r e c}(x) \mathrm{d} x}{1+\lambda_{e} B \lambda_{s} \pi(R(\theta))^{2}} .
$$

One can prove (see [8]) that the policy

$$
d(x)=\mathbf{1}\left(|x|<R\left(\underline{\theta}^{*}\right)\right)
$$

maximizes $\underline{U}$ (see Figure $[5$ ), (right). 


\section{Concluding remarks and future work}

In this paper we presented a detailed mathematical model of the physical and medium access layer for a non-slotted Aloha channel without feedback. As the main result we proved an analog of the Erlang's formula for the rate of the packets that are successfully received in this channel. The size of the packet is assumed for simplicity to be constant, but our approach can be extended to a variable packet size, which is natural for the non-slotted channel. Taking into account the retransmissions (stipulated by Aloha) is more tricky. One knows that any "infinite population" model (as our Poisson rain) with exponential back-off is instable.

As an example of application, we demonstrated how this model can be used to optimized the sensor-to-sink transport is some sensor network. We were interested in maximizing the coverage of some sensing domain or in increasing the total throughput. More systematic approach is possible on the ground of the fairness and control theory. In particular, the two contradictory goals considered in Section 5.3 are realized by the policies respectively called max-min and globally optimal. We did not discuss implementation details of the optimal policies. One example is considered in [9]. For simplicity we considered only policies based on mean received power (which is equivalent to distance-based policies). Analysis of more opportunistic policies, based on instantaneous received powers are left for future work. Also, we considered only one cluster head scenario. We leave for future work analysis and optimization of more complex architectures, which may motivate the deployment of such sensor networks in which some sensors are cheaper and less energy consuming, because they do not have receiver circuit.

\section{Acknowledgment}

The authors are grateful to François Baccelli for many helpful discussions and to Serguei Foss for pointing out some more general perspectives for the model.

\section{References}

[1] R. Wolff, Stochastic Modeling and the Theory of Queues. Prentice-Hall, 1989.

[2] D. Bertsekas and R. Gallager, Data Networks. London: Prentice-Hall, 1987.

[3] A. Akyildiz, "ESRT: Event-to-sink reliable transport in wireless sensor networks," in Proc. of ACM Mobihoc, 2003.

[4] D. Tse and P. Viswanath, Foundamentals of Wireless Communication. Cambridge University Press, 2005.

[5] F. Baccelli and P. Brémaud, Elements of Queueing Theory, ser. Série: Applications of Mathematics. Springer Verlag, 2002, second edition. 
[6] D. Stoyan, W. Kendall, and J. Mecke, Stochastic Geometry and its Applications. Chichester: John Wiley and Sons, 1995.

[7] S. Zuyev, Strong Markov Property of Poisson Processes and Slivnyak Formula, ser. Lecture Notes in Statistics. Springer, 2006, vol. 185, pp. 77-84.

[8] B. Błaszczyszyn and B. Radunović, "On performance of event-to-sink transport in transmit-only sensor networks," 2006. [Online]. Available: https://hal.inria.fr/inria-00092002

[9] B. Radunović, H. L. Truong, and M. Weisenhorn, "Receiver architectures for transmitonly, UWB-based sensor networks," 2005. 


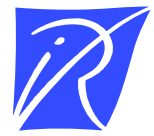

Unité de recherche INRIA Rocquencourt

Domaine de Voluceau - Rocquencourt - BP 105 - 78153 Le Chesnay Cedex (France)

Unité de recherche INRIA Futurs : Parc Club Orsay Université - ZAC des Vignes

4, rue Jacques Monod - 91893 ORSAY Cedex (France)

Unité de recherche INRIA Lorraine : LORIA, Technopôle de Nancy-Brabois - Campus scientifique

615, rue du Jardin Botanique - BP 101 - 54602 Villers-lès-Nancy Cedex (France)

Unité de recherche INRIA Rennes : IRISA, Campus universitaire de Beaulieu - 35042 Rennes Cedex (France)

Unité de recherche INRIA Rhône-Alpes : 655, avenue de l'Europe - 38334 Montbonnot Saint-Ismier (France)

Unité de recherche INRIA Sophia Antipolis : 2004, route des Lucioles - BP 93 - 06902 Sophia Antipolis Cedex (France)

INRIA - Domaine de Voluceau - Rocquencourt, BP 105 - 78153 Le Chesnay Cedex (France)

http://www.inria.fr

ISSN 0249-6399 